\title{
ON ALGEBRAIC EQUATIONS HAVING ONLY REAL ROOTS*
}

\author{
BY W. E. ROTH
}

Given the algebraic equation

(1) $f_{1}(x)=x^{n}+a_{1} x^{n-1}+a_{2} x^{n-2}+\cdots+a_{n}=0, a_{n} \neq 0$,

whose roots $x_{i}, i=1,2, \cdots, n$, are all real. Then there exist equations, $f_{\lambda}(x)=0$, also of degree $n$, whose roots are the real numbers $x_{i}{ }^{\lambda},(i=1,2, \cdots, n ; \lambda= \pm 1, \pm 2, \cdots)$. If

$$
S_{j}=x_{1}{ }^{j}+x_{2}{ }^{j}+\cdots+x_{n}^{j}, \quad(j=0, \pm 1, \pm 2, \cdots),
$$

then the determinants

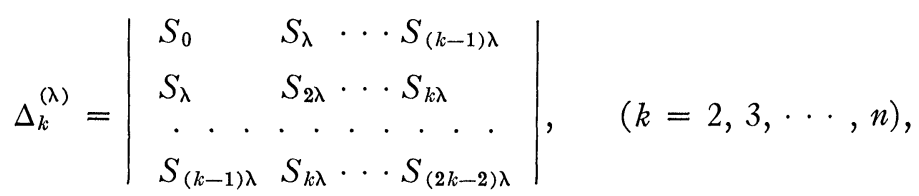

are all positive according to Borchardt's theorem $†$ provided the roots of $f_{\lambda}(x)=0$ are distinct. On the other hand, if $f_{1}(x)=0$ has exactly $\mu(\mu \leqq n)$ distinct roots, all real, then for odd values of $\lambda, f_{\lambda}(x)=0$ will have exactly the same number of distinct roots; and for even values of $\lambda$, exactly $\mu-\nu$ distinct roots, where $\nu$ is the number of distinct pairs of numerically equal roots of $f_{1}(x)=0$ which differ only in sign. Under these hypotheses, it is known that

$$
\begin{array}{rrr}
\Delta_{k}^{(\lambda)}>0, & (k=2,3, \cdots, \mu), \\
=0, & (k=\mu+1, \mu+2, \cdots, n),
\end{array}
$$

if $\lambda$ is an odd positive or negative integer, and that

$$
\begin{array}{rr}
\Delta_{k}^{(\lambda)} & >0, \quad(k=2,3, \cdots, \mu-\nu), \\
& =0, \quad(k=\mu-\nu+1, \mu-\nu+2, \cdots, n),
\end{array}
$$

* Presented to the Society, November 28, 1931.

$\dagger$ Borchardt, Journal de Mathématiques, vol. 12 (1847), p. 58; Werke, Berlin, (1888), p. 24. 
if $\lambda$ is an even positive or negative integer. ${ }^{*}$ Upon the basis of the above known results we shall establish the following theorem and shall develop some of its implications.

THEOREM. If the roots $x_{i},(i=1,2, \cdots, n)$, of the equation

$$
f_{1}(x)=x^{n}+a_{1} x^{n-1}+a_{2} x^{n-2}+\cdots+a_{n}=0, a_{n} \neq 0,
$$

are all real and if we use the notation (2), then all roots of $f_{1}(x)=0$, whose multiplicity equals or exceeds $r$, must lie on the real intervals where the following inequalities in $x$ hold:

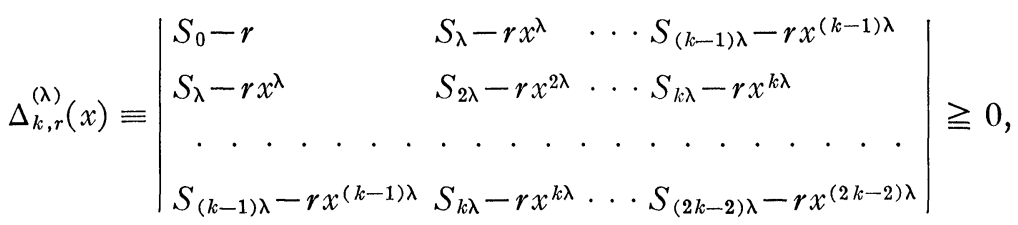

$$
\begin{aligned}
& (k=2,3, \cdots, n-r+2) .
\end{aligned}
$$

Let $x_{i}$ be any root of $f_{1}(x)=0$ whose multiplicity equals or exceeds $r$, then there exist equations

$$
F_{i, r}^{(\lambda)}(x) \equiv \frac{f_{\lambda}(x)}{\left(x-x_{i}{ }^{\lambda}\right)^{r}}=0, \quad(\lambda= \pm 1, \pm 2, \cdots),
$$

for $i=1,2, \cdots, \mu$, of degree $n-r$ in $x$, whose roots are identical with those of $f_{\lambda}(x)=0$ save that the multiplicity of the root, $x^{\lambda}$, is reduced by $r$. The sum of the $j$ th powers of the roots of (3) is then given by $S_{j \lambda}-r x_{i}{ }^{j}$, where $S_{j \lambda}$ is given by (2). Now the roots of $F_{i, r}(x)=0$ are all real, hence the first principal minors of its discriminant are $\Delta_{k, r}^{(\lambda)}\left(x_{i}\right),(k=2,3, \cdots, n-r)$, and all these minors, where $r$ does not exceed the multiplicity of the root, $x_{i}$, of $f_{1}(x)=0$, are positive or zero. If $f_{\lambda}(x)=0$ has $\mu$ distinct roots $x_{i}{ }^{\lambda},(i=1,2, \cdots, \mu)$, of multiplicity $r_{i}$, respectively, then we can readily show that

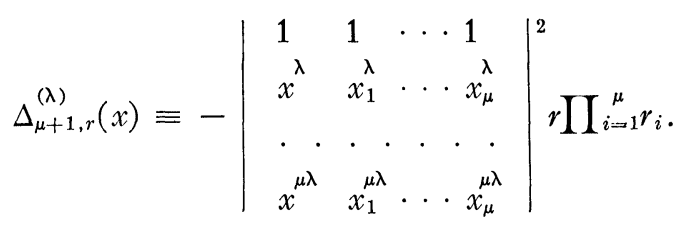

* Baur, Mathematische Annalen, vol. 50 (1898), p. 241. Grommer, Journal für Mathematik, vol. 144 (1914), p. 114. 
Hence $\Delta_{\mu+1, r}^{(\lambda)}(x)=0$ only for values of $x$ such that $f_{1}(x)=0$ and is negative for all other values of $x$, whatever $r$ may be. Thus the equality sign given in the theorem above holds in this particular case. If $\mu=n$ we find, as a direct consequence of (4), that

$$
\Delta_{n+1,1}^{(\lambda)}(x) \equiv f_{1}^{2}(x) K(x),
$$

where $K(x)$ is a polynomial in $x$. For $k \geqq \mu+2$

$$
\Delta_{k, r}^{(\lambda)}(x) \equiv 0
$$

as can be readily verified by expanding this determinant in powers of $x^{\lambda}$. All coefficients of the expansion are the sums of minors of order $k-1$ of $\Delta_{k, 0}^{(\lambda)}(x)$, and are consequently zeros. That is, if $k$ exceeds by two or more the number of distinct roots of $f_{\lambda}(x)=0, \Delta_{k, r}^{(\lambda)}(x)$ is identically zero and the theorem is satisfied. An additional special case of interest is the following. If $\mu=n$ or $n-1$,

$$
\Delta_{n, 1}^{(\lambda)}(x) \equiv f_{1}(x) L(x),
$$

where $L(x)$ is a polynomial in $x$. If $\mu=n-1$, this identity holds because of (4). Therefore we shall assume that $\mu=n$. Evidently

$$
\Delta_{n, 1}^{(\lambda)}\left(x_{i}\right)=0, \quad(i=1,2, \cdots, n) ;
$$

since this is a determinant of order $n$ in the elementary symmetric functions of the $n-1$ roots of the equation $F_{i, r}^{(\lambda)}(x)=0$, $(i=1,2, \cdots, n)$, respectively, and the identity above is proved. Hence we have shown that $\Delta_{k, r}^{(\lambda)}(x)$ must be positive or zero on any interval which contains a root of $f_{1}(x)=0$ whose multiplicity equals or exceeds $r$.

We shall now prove a generalization of a theorem due to Laguerre* as a corollary of the above theorem.

Corolla Ry 1 . If $f_{1}(x)=0$ has the real roots $x_{i},(i=1,2, \cdots, n)$, not necessarily all distinct, but $f(0) \neq 0$, and if we use the notation (2), then all roots of $f_{1}(x)=0$ whose multiplicity equals or exceeds $r$, must lie on the interval where the inequalities

* Laguerre, Sur une méthode pour obtenir par approximation les racines d'une équation algébrique qui a toutes ses racines réelles, Nouvelles Annales de Mathématiques, (2), vol. 19 (1880), pp. 161-171; see particularly the footnote on page 169. See also Kraus, Časopis pro Pěstování Mathematiky a Fysiky, vol. 15 (1886), p. 63. 


$$
\begin{aligned}
& \frac{r S_{\lambda}-\left[r(n-r)\left(n S_{2 \lambda}-S_{\lambda}^{2}\right)\right]^{1 / 2}}{n r} \leqq x^{\lambda} \\
& \leqq r S_{\lambda}+\left[r(n-r)\left(n S_{2 \lambda}-S_{\lambda}^{2}\right)\right]^{1 / 2} \\
& n r
\end{aligned}
$$

$(\lambda= \pm 1, \pm 2, \cdots)$, hold for real $x$.

This corollary follows directly from the inequality $\Delta_{2 . r}^{(\lambda)}(x) \geqq 0$. In this as in (5) the equality signs hold only if $f_{\lambda}(x)=0$ has an $r$-fold root and at most one $s$-fold root distinct from it. The radical in (5) is always real because $n S_{2 \lambda}-S_{\lambda}{ }^{2}$ is the first principal minor of order two of the discriminant of $f_{\lambda}(x)=0$, and is positive. The limits between which all roots whose multiplicity equals or exceeds $r$ must lie, according to the corollary above, are expressed in terms of the $2 \lambda$ coefficients $a_{1}, a_{2}, \cdots, a_{2 \lambda}$ of $f_{1}(x)$ in case $\lambda$ is a positive integer and in terms of the $1-2 \lambda$ coefficients, $a_{n+2 \lambda}, a_{n+2 \lambda-1}, \cdots, a_{n}$, in case $\lambda$ is a negative integer. We shall now show that the intervals where roots of $f_{1}(x)=0$ must lie are reduced as $\lambda$ is increased in numerical value. If $\alpha$ is the absolute value of the numerically greatest root of $f_{1}(x)=0$, then

$$
\frac{S_{\lambda}}{\alpha^{\lambda}}=\left(\frac{x_{1}}{\alpha}\right)^{\lambda}+\left(\frac{x_{2}}{\alpha}\right)^{\lambda}+\cdots+\left(\frac{x_{n}}{\alpha}\right)^{\lambda}=\tau_{1}+a, \quad\left(\left|\tau_{1}\right|<n\right),
$$

where $\tau_{1}$ is a positive or negative integer giving the sum of the terms in which $\left|x_{i}\right|=\alpha$, and $a$ is the sum of the remaining terms of the right member in which $\left|x_{i}\right|<\alpha$. In the following we shall assume that $\tau_{1}>0$. Similarly

$$
S_{2 \lambda} / \alpha_{2 \lambda}=\tau_{2}+b>0, \quad\left(n>\tau_{2}>0\right),
$$

where $\tau_{2}$ is the sum of the terms in which $\left|x_{i}\right|=\alpha$ and $b$ is the sum of those in which $\left|x_{i}\right|<\alpha$. If $\lambda$ is even, $\tau_{1}=\tau_{2}$. By taking $\lambda$ sufficiently large $a$ and $b$ can both be made as small as we please for each $\left(x_{i} / \alpha\right)^{\lambda},\left(x_{i}<\alpha\right)$, approaches zero as $\lambda$ grows. From (5) we get

$$
\left(\frac{x}{\alpha}\right)^{\lambda} \leqq \frac{\tau_{1}+a}{n}+\left\{\frac{n-r}{r}\left[\frac{\tau_{2}+b}{n}-\left(\frac{\tau_{1}+a}{n}\right)^{2}\right]\right\}^{1 / 2} .
$$


Now $n S_{2 \lambda} \geqq S_{\lambda}^{2}$; hence we have

$$
\frac{\tau_{2}+b}{n} \geqq\left(\frac{\tau_{1}+a}{n}\right)^{2}
$$

But for positive $\lambda,\left(\tau_{2}+b\right) / n<1$; hence

$$
1>\frac{\tau_{2}+b}{n} \geqq\left(\frac{\tau_{1}+a}{n}\right)^{2},
$$

or

$$
1>1-\left(\frac{\tau_{1}+a}{n}\right)^{2}>\frac{\tau_{2}+b}{n}-\left(\frac{\tau_{1}+a}{n}\right)^{2} \geqq 0 ;
$$

that is $\left(\tau_{1}+a\right) / n<1$, if $\tau_{1}>0$. Then

and

$$
\left(\frac{x}{\alpha}\right)^{\lambda} \leqq 1+\left(\frac{n-r}{r}\right)^{1 / 2} \leqq 1+(n-1)^{1 / 2},
$$

$$
x \leqq\left(1+(n-1)^{1 / 2}\right)^{1 / \lambda} \alpha .
$$

The coefficient of $\alpha$ approaches unity as $\lambda$ increases. Similarly if $\tau_{1}<0$ and $\lambda$ is an odd positive in teger,

$$
x \geqq-\left[1+(n-1)^{1 / 2}\right]^{1 / \lambda} \alpha .
$$

Hence the numerically greater bound obtained from (5) when $\lambda$ is positive approaches the root of greatest numerical value as $\lambda$ is given larger and larger integral values. Similarly the numerically lesser bound given by (5) for negative values of $\lambda$ approaches the root of least numerical value as $-\lambda$ is given ever larger integral values.

The theorem of Laguerre mentioned above is obtained from our corollary by letting $\lambda=1$ and $r=1$. Laguerre gave two proofs of his theorem either of which, by the use of the polynomial $f_{\lambda}(x)$, could have been extended to cover the case $\lambda>1$. This obvious extension seems to have escaped later writers who proved the theorem. A generalization of the theorem was made by Pleskot; its proof rests upon the inequality

* Pleskot, Über die Grenzen der Wurzeln einer Gleichung mit nur reellen Wurzeln, Sitzungsberichte der Böhmischen Gesellschaft der Wissenschaften in Prag, 1897, No. 37; Limites des racines d'une équation n'ayant que des racines réelles, Nouvelles Annales de Mathématiques, (3), vol. 18 (1899), pp. 301-305. 


$$
\frac{S_{2 \lambda}-x^{2 \lambda}}{n-1} \geqq\left(\frac{S_{1}-x}{n-1}\right)^{2 \lambda}, \quad(\lambda=+1,2, \cdots),
$$

which he proved. However, since $\Delta_{2,1}^{(2 p)}(x) \geqq 0$, we have at once the inequality

$$
\frac{S_{4 p}-x^{4 p}}{n-1} \geqq\left(\frac{S_{2 p}-x^{2 p}}{n-1}\right)^{2},
$$

which is only another way of writing (5) when $\lambda=2 p$. From this and Pleskot's inequality we have

$$
\frac{S_{4 p}-x^{4 p}}{n-1} \geqq\left(\frac{S_{2 p}-x^{2 p}}{n-1}\right)^{2} \geqq\left(\frac{S_{1}-x}{n-1}\right)^{4 p} .
$$

Pleskot would use the first and last members of this inequality to compute his bounds and above in (5) we use the first two members, hence for even values of $\lambda$ we get at least as good bounds for the roots as does Pleskot. If $\lambda$ is an odd integer the same conclusion may be valid but has not been proved by the writer. Take the equation

$$
f_{1}(x)=(x+4)(x+1)(x-2)(x-3)=0
$$

as an example to illustrate the latter case. Pleskot's inequality

$$
\frac{S_{6}-x^{6}}{n-1} \geqq\left(\frac{S_{1}-x}{n-1}\right)^{6}
$$

gives us the limits

$$
-4.117 \leqq x \leqq 4.117
$$

and

$$
\frac{S_{6}-x^{6}}{n-1} \geqq\left(\frac{S_{3}-x^{3}}{n-1}\right)^{2},
$$

which is (5), for $\lambda=3, r=1$, gives us $-4.055 \leqq x \leqq 3.725$. Both limits are nearer the extreme roots of the given equation in the latter than in the former inequality.

Nagy* gives another demonstration of Laguerre's theorem, that is, of Corollary 1 for the case $\lambda=1, r=1$, which he bases upon a theorem by Schur.

* Nagy, Über algebraische Gleichungen mit lauter reellen Wurzeln, Jahresbericht der deutschen Mathematiker Vereinigung, vol. 27 (1918), pp. 37-45. 
Corollary 2. If $\lambda$ is an odd integer and if $S_{\lambda}{ }^{2}>(n-r) S_{2 \lambda}$, then all roots of $f_{1}(x)=0$ whose multiplicity equals or exceeds $r$ are positive or all are negative according as $S_{\lambda}$ is positive or negative.

This proposition follows at once from Corollary 1 . When it is satisfied the relationship (5) provides upper and lower bounds for the roots in question whether $\lambda$ be positive or negative. However, if $f_{1}(x)=0$ has both positive and negative $r$-fold roots, then $S_{\lambda}{ }^{2}<(n-r) S_{2 \lambda}$, where $\lambda$ is an odd positive or negative integer; and in this case (5) provides us with an upper bound for the negative and a lower bound for the positive $r$-fold roots if $\lambda$ is negative and extreme bounds for all $r$-fold roots if $\lambda$ is positive.

It is easily shown that all principal minors of $\Delta_{n, r}^{(\lambda)}(x)$ are positive or zero on intervals containing $r$-fold roots of $f_{1}(x)=0$. Hence $S_{2 \lambda}-r x^{2 \lambda}>0, \quad(\lambda= \pm 1, \pm 2, \cdots)$, on any interval where $r$-fold roots of $f_{1}(x)=0$ lie.

A great variety of formulas bounding the roots of $f_{1}(x)=0$ can be based on other principal minors of $\Delta_{n, r}^{(\lambda)}(x)$ than those employed here and in Corollary 1 , but they require the solution of equations of degrees higher than the second in $x^{\lambda}$, and are therefore not readily solved for the limiting values.

Finally we can show that

$$
\frac{S_{ \pm(2 \lambda+2)}}{S_{ \pm 2 \lambda}}<x^{ \pm 2},
$$

where $\lambda$ is positive and the subscripts and exponents are either all positive or all negative. This inequality gives a lower bound of the numerical value of the largest numerical root if the positive signs are taken, and an upper bound to the numerical value of the least numerical root if the negative signs are taken. The relation is a special case under the Lagrange-Bernoulli theorem.*

UNIVERSITY OF Wisconsin, Extension Division

* Pascal's Repertorium, vol. I, 1, 1910, p. 355. 